\title{
Abstract
}

This paper presents findings from a qualitative case study that explored long term integration of internationally educated nurses in an Ontario healthcare facility. Using critical social theory as the philosophical underpinnings for this research, we selected the case based on the hospital's history of employing and supporting internationally educated professionals. Data sources included: documents review, twenty-eight interviews, socio-demographic survey and five focus groups involving IENs and other stakeholders. An overarching theme points to a 'two-way' notion of workplace integration whereby efforts are required on the part of the employer as well as the IENs. An in-depth analysis of the data reveals sub-processes of two-way integration: respecting diversity and difference, adopting inclusive practices and striving to achieve equity. Challenges in achieving two-way integration are discussed. Implications for nursing leaders to tap into IENs' diverse talents for the benefit of their local healthcare systems are highlighted.

Keywords equity, internationally educated nurses, inclusive practices, two-way integration, workplace integration

\section{Unpacking "two-way" workplace integration of internationally educated nurses}

\section{ZUBEIDA RAMJI, JOSEPHINE ETOWA \\ \& ISABELLE ST-PIERRE}

\section{Introduction}

For over a decade now, internationally educated nurses (IENs) as a specific segment of the nursing workforce have emerged as a focus for research. Most of this research examines the experiences, challenges and supports for IENs as they migrate to their new country context, navigate through processes of credentials assessments and registration, find meaningful employment and transition into nursing workplaces.[1-6] The nursing literature appears one-sided as it only emphasizes what is required for IENs to adjust to the host environment.[7] While the term "workplace integration" is frequently used about IENs, a definition is not provided.[8] In addition, literature on the experience of IENs over their long-term, post-transition phase[9-11] is scarce. The ways in which the organizational context influences IENs' integration in the workplace has not been a clear research focus. $[7,9]$

This research addresses the gaps by focusing on the experiences, achievements and contributions of IENs who are beyond transition and have been nursing in Canada for an average of eleven years. Early adaptation efforts towards a successful transition phase are critical in ensuring that IENs have grasped the differences in the role and scope in their nursing practice and are meeting the employer's probationary requirements.[6] However, the longer-term focus is necessary to understand IENs' ongoing satisfaction, progress and retention within the workplace and nursing profession.[12,13] This research's emphasis on the organizational context explores workplace integration as a 'two-way' process in nursing and generates insights for how 
employers can effectively facilitate workplace integration of IENs.

\section{Purpose}

This paper is based on a study that aimed at understanding workplace integration from perspectives of both IENs and other stakeholders. The research was guided by concepts borrowed from social sciences about societal integration of immigrants and refugees, observations about healthcare employers' approaches to organization-wide efforts to support IENs, and the authors' own experiences.

This paper explores the overarching theme of workplace integration of IENs as a 'two-way' process and outcome. The notion of two-way integration is unpacked to outline three sub-processes: (1) Respecting diversity and difference, (2) Adopting inclusive practices and (3) Striving to achieve equity. The various levels of commitment and efforts required by the IENs as well as the employer organization to facilitate integration are highlighted, along with discussion of the major challenges encountered. The paper situates these findings within existing literature while highlighting implications for nursing and healthcare.

\section{Theoretical Perspectives}

Critical social theory (CST) provides relevant philosophical underpinnings for this research on workplace integration of IENs. While works of various theorists and thought leaders were considered, Lukes' [14] three dimensions or faces of power was found to be especially relevant in terms of how oppression and power imbalances impact not only IENs but also the structures and systems that make up their workplace organizations.

Lukes' first dimension or overt face of power is the pluralists' perspective about the way decisions are made: "A has power over $B$ to the extent that he can get $B$ to do something that B would not otherwise do".[15 p5] In this dimension the focus is on who participates, who gains and who loses, and who prevails in decision-making.[14-16] The assumption is that the decision-making process is open to everyone or virtually any organized group who cares about the issues at hand.[14-16] Since the process is considered open, leaders are assumed to be speaking on behalf of a group and so nonparticipation or inaction is not seen as a problem but is taken to reflect consensus.[14-16] With respect to marginalized groups, in this dimension, the possibility of power relations is not considered but instead non-participants are blamed or thought of as apathetic or incompetent.[14-16] Strategies to correct this situation try to "educate" or change the nonparticipants, still with the assumption that power constraints will not pose any barriers to participation. [14-16]

The second dimension or covert face of power excludes certain participants and issues altogether. This dimension creates bias by establishing what is important and what is unimportant. Organizations or groups take advantage of certain kinds of conflict and actively suppress others, so that issues are prevented from arising in the first place.[14-16)] The focus is not to prevail in a struggle, but to predetermine the agenda of the struggle itself.[14-16] This dimension does not consider how power intervenes in the issue-raising process and that fear and vulnerability might explain nonparticipation by marginalized groups. [14-16]

The third dimension or latent face of power was developed by Lukes[14] as follows: "A exercises power over B by getting her to do what he does not want to do, but he also exercises power over her by influencing, shaping or determining her very wants".[15 p12] In other words, the powerful group prevents the marginalized from effectively raising issues which also impacts their conception of the issues altogether.[14-17] This dimension is the hardest of all to identify because people influenced by this latent power find it difficult to discover its very existence.[14-17]

A CST perspective informed by theorists such as Lukes[14] ensures that organizations are taking on their share of responsibility to shift power and dismantle barriers, by questioning and analyzing all structures and practices. Various studies about racism in nursing have carried out analyses of how organizational policies and structures embedded with dominant white norms and values have been problematic for Black[18,19] and immigrant nurses.[20] A similar critical analysis appears to be absent in the body of literature about IENs. This research adds such critical analysis of power and its potential effects on IENs in the workplace.

\section{Methodology}

This qualitative research used an instrumental case study approach as described by Stake,[21] whereby the case organization facilitates the understanding of the phenomenon of interest. St. Michael's Hospital (SMH) in Toronto, Canada, with a track record of supporting IENs[22] was selected as the case allowing a critical examination of how integration of IENs unfolds through organizational initiatives. Data collection took place between October 2014 and March 2015 after obtaining approval from the research ethics boards of SMH and University of Ottawa. The sample of 28 
participants was diverse with respect to age range, gender, country of origin and nursing education, ethno-racial heritage, immigration status and number of years and types of nursing or other professional work experiences. Fifty percent were IENs and the rest of the participants included peers/mentors (18\%), managers/directors (21\%) and senior leaders (11\%). Participants were accessed through recruitment strategies such as presentations at meetings, information letters and posters. Purposeful sampling was carried out first, followed by snowballing technique to ensure diversity of IENs and others in the sample.

Four forms of data collection were used: semi-structured interviews; socio-demographic survey; focus groups and review of organizational documents such as strategic plans, project reports, health equity plans/reports, in order to understand the context of the stated commitment to supports for IENs. At the focus groups, a presentation of preliminary analysis preceded the discussion and had the added purpose of serving as a form of member checking and involving participants in shaping study recommendations. Informed consent was obtained from each participant. At the organizational level, permission was obtained from senior representatives about identification of the study site during and/or after completion of the research. Privacy and confidentiality was maintained by giving participants the option of being interviewed at an accessible offsite location away from their area of work; alpha-numeric code numbers were used to identify participants; interview notes, demographic profile and consent forms were separated from the data files and all electronic data files and storage devices were password protected. In reporting out the findings, quotes were selected carefully so as not to inadvertently identify the participants.

Thematic analysis was carried out in various stages. At a preliminary level, data analysis occurred simultaneously with data collection; it was inductive and iterative. Inductive coding of the data did not start with a pre-established list of codes but instead the codes emerged from the data.[23] After analyzing perspectives of each individual participant (within subcase analysis), the data was then re-sorted using NVivo 10 according to four stakeholder groups that make up the sample (i.e. between sub-unit analysis - of IENs, peers/mentors, managers/directors and senior managers). This was followed by analysis of IENs across each of the sub-units, namely: peers/mentors, managers/directors and senior managers - to identify areas of convergence and divergence.[21]

\section{Finding: Two-way Integration of IENs}

The overarching finding in this case study is that workplace integration of IENs occurs through a two-way process, requiring efforts from both the IEN and organization. Twoway integration has been categorized into three main sub-processes; (1) Respecting diversity and difference, (2) Adopting inclusive practices, and (3). Striving to achieve equity. Key messages conveyed in each of the sub-processes overlap but there are varying emphases in terms of ongoing changes and efforts required on the parts of the IEN and the workplace organization.

\section{Respecting diversity and difference.}

Respecting diversity and difference is demonstrated by IENs and the workplace organization in the way the needs of an increasingly diverse patient base are addressed. For example, the organization values how the diverse backgrounds of IENs help address patient diversity, but also acknowledges that IENs might experience various challenges in their transition from their former practice environments. IENs recognize and accept the responsibility of adapting to the numerous differences in nurses' role in Canada, along with grasping complexities of working with diverse patients and colleagues.

To adapt and make the transition into their new practice environment, IENs must invest time and resources to learn about nursing in Canada. When integrated, IENs are confident in their understanding of how the legal and professional frameworks that govern the role of the nurse are different from those in the other jurisdictions where they practiced. The context of being immersed in inter-professional teams and specifically their level of professional autonomy with regards to their interaction with physicians, is a major adjustment for most IENs. The differences in the role and scope of nurses' practices are described by an IEN:

[Nurses] have a lot more autonomy and they're expected to ...like we'll get the doctors asking us, well, what do you think? ...they trust you as a professional to make decisions ...your scope of practice is actually bigger here ... (participant I001)

Aside from learning cultural nuances of behaviours of their diverse patients, colleagues and team members, many IENs have had the added challenge of mastering 'Canadian' English: "When I was new, they said I don't speak English well. They questioned my skills. As if they don't trust me..." (participant 1017). This can be a major hurdle for many IENs, even those who perceived themselves as having come to Canada with adequate English language skills. This is further reinforced by a manager who said: 
...it is one thing to be able to speak English but it's another thing to get your point across in a way that's clear and that doesn't, you know, perpetuate stereotypes of an internationally educated person being not so educated (participant L005).

Once the IEN is integrated, s/he is seen to be 'fitting in' with the team and is valued by co-workers. The place of where $\mathrm{s}$ /he obtained the basic nursing education is not relevant anymore. One manager states: "The IENs that I work with, if I didn't know that they [had] practiced elsewhere or they got trained in a foreign country...now, I wouldn't know" (participant M004). Overall, the IEN has a greater sense of belonging when integrated. A manager explains how fellow colleagues in the workplace environment need to be open and willing to embrace IENs:

And again, that's a two-way street. So the people who are within the hospital have to be ready and willing to accept someone who... may be on a learning curve in terms of culture and linguistics and practices and Canadian nursing practice (participant L005).

At the organizational level, a strong commitment to patients is evident in explicit statements of values and beliefs about respect for diversity and difference. There is recognition that having a workforce reflective of the ethno-racial and linguistic diversity within the patient population is important to improve access to services and supports. Workforce diversity is a priority according to this IEN:

It's the values of St. Mike's...we work with the very marginalized population, very diverse and very challenging. And to do the best job, you have to have [a] diverse workforce. And what's a better way than to have diversity in your nurses (participant 1027).

The diversity within the workforce reinforces the sense of belonging. This is described by IENs as a key factor in how the context enables workplace integration:

We're really like a multicultural unit... I like it, because again, it's like we learn things from each other and you know, it's great because we've got a nurse who speaks Mandarin, one who speaks Cantonese, one speaks Vietnamese, one speaks Korean... three of them speak Portuguese. So it's great, we have people who can help each other out and like it's really quite fun (participant I001).

Managers emphasize the need to help IENs adapt to the new practice environment and become 'Canadian nurses'. Concern is shown for ensuring that there is equality of treatment for IENs.

This first sub-process of two-way integration focused on respecting diversity and difference, stems from the employer's commitment to its increasingly diverse patient base. IENs are a valuable resource for diversifying the nursing workforce and for reducing language or cultural barriers for patients and families. IENs have gone through an intense period of learning and relearning to adapt to Canadian nursing practice and to fit into their workplace. However, there are challenges when despite the growing diversity in the workforce, the policies and procedures are based on the assumptions and beliefs of the dominant group. This leads us to the next section where two-way integration is explained as a process focused on adopting inclusive practices.

\section{Adopting inclusive practices}

This sub-process of two-way integration emphasizes the workplace's commitment to an active stance in addressing exclusion experienced by anyone who, because of their differences, may be at risk of marginalization. Building on the context of a highly diverse workforce, systematic approaches to creating inclusive policies and practices can change the experience of staff, patients and the broader community. At the individual level, IENs become sensitized, accept the need to change their outlook and embrace inclusive practices themselves.

$\mathrm{SMH}$, the case organization, has incorporated its commitment to creating and sustaining an inclusive organization in its mission, values and strategic plan. This commitment is enacted through various initiatives. A senior manager explains how the right to being different is embedded in the inclusive workplace culture:

We have a culture...that is very open and inclusive to patients, if you like... that is very acceptant of different ways of talking and different ways of being. There's a non-judgmental sort of approach to care. Almost in the extreme ...people behaving in ways that are totally objectionable and we still are welcoming and open. So if you can do that with patients, you can do that with each other, right...People are looking out for you as opposed to... judging you (participant L024).

Specific roles such as the Diversity and Special Projects Coordinator or more recently, the Director of Mission and Values have been in place to promote $\mathrm{SMH}$ 's values of respect and inclusion of all peoples. An annual awards program which celebrates staff who have excelled at demonstrating the organization's mission and values, creates deliberate hype within the workplace to reinforce this priority. Additionally, the corporate orientation program for all new staff dedicates time for training about diversity, inclusion and equity.

Being aware of cultural differences and accepting diverse value systems are described by this senior manager as part of the integration process for IENs at $\mathrm{SMH}$ :

I remember from my days, [when I was] responsible for the obstetrics unit and we had lots of nurses who were very judgmental towards some of our street folks. Particularly, street workers who were in for some reason usually of a pregnancy, delivery or whatever... the manager had to be very sensitive about the fact that this is different than from the Philippines and 
there are different norms and values and expectations (participant L025).

Immersion within such a workplace context over the long term has transformative effects on IENs as noted by this IEN:

Back home, we seldom work with different [diverse] people...but then [once] you have integrated, you don't look that they're different...you're not racist... you respect that regardless of their backgrounds... cultural or sexual orientation, religious background... talking about St. Mike's, because we have diverse people here (participant I017).

The exposure to co-workers and patients of diverse backgrounds allows the integrated IEN to re-evaluate her/his own values/beliefs. One IEN explains:

When you're an IEN, you come from somewhere, you come with several different cultures and values that you want to integrate with...some you will drop by the side, some you will redefine, and some you will refine. You know, it's all part of the integration (participant I012).

Aside from valuing diversity and differences that IENs bring to the workforce, senior level champions recognize need for inclusive practices that facilitate their integration. A strategic management approach whereby the priority is championed is discussed by this leader:

Strategically and philosophically as a leader, I have to see the value of our IENs to our organization and to our healthcare system. So, if I don't see that, I'm not going to really invest any time or energy in that. So that's why I see my role as an executive leader to make sure that my other executive colleagues understand this is our commitment and support it and also that I'm working with directors and managers (participant L018).

Leaders understand that the 'one size fits all' approach to workplace integration does not work. Nurse managers' encouraging and supportive style which recognizes the skills and expertise that IENs are bringing, is seen to be critical to their successful integration. A manager explains:

I need to buddy you up with somebody, who is the best person, who am I going to buddy you up with regards to making you succeed and making you feel comfortable and making you shine or blossom or open up and we see the true potential of what you can provide (participant L003).

In this research, two policy areas were highlighted as affecting IENs differently than perhaps many of their peers: dealing with intolerant and racist behaviours and the use of one's mother tongue (other than English) in the workplace.

The effects of intolerant and racist behaviours of patients and/ or their family members is described by this IEN:

They look at your skin, they say, oh, I don't want to be treated by a brown skinned or a black skinned nurse. I want to be treated by [a] "Canadian", [implying] a white skinned...It's really [difficult], sometimes you feel disrespected (participant I013).

$\mathrm{SMH}$ has given priority and invested resources towards organization-wide implementation of best practice guidelines in order that all affected individuals, bystander colleagues and managers respond appropriately to intolerance or racism. The importance of consistent application of the protocols is described by this IEN:

Sometimes they [patients/families] really use some bad words to us... but luckily we have other staff, they help us to talk to the patient...or take over from us... the manager comes and gets involved and talks to the patient... (participant I021).

The issue of staff members' use of their mother tongue (other than English) emerged as another policy area requiring a more balanced view. Managers recognize the value of a diverse staff team, including the camaraderie between IENs and other nurses who are of a similar heritage. At the same time, there is a firm policy that English is the language of the workplace to ensure inclusion of the entire team. A manager states:

The expectation is that you speak English...so when you are practicing, when you're away from the unit, people are free to speak their mother tongue but when you're in a work environment and you're in a team, the expectation is that you're speaking English because that is the common language that everybody speaks (participant L009).

When it comes to caring for patients and families from backgrounds like their own, IENs are seen to have a direct benefit on how clinical assessments and/or treatments are facilitated, resulting in shorter lengths of stay, lower costs in terms of physician time and interpretation services. Managers/ directors agree that lack of formal acknowledgment of the value of IENs' multi-lingual skills is a contradiction and source of tension, which should be addressed by restating related policies. The cumulative effects of a responsive and inclusive context result in the integrated IEN feeling more at ease and comfortable about her/his cultural identity. One IEN explains:

So in my mind being really well integrated means you are able to accept the culture of this country and of the workplace but you're also able to own your own culture that you brought with yourself. (participant I027)

Central to this second sub-process of two-way integration is the leadership's commitment to creating and sustaining an inclusive environment. This commitment is based on the belief that people have a right to be different and that 
the organization has a responsibility to adjust its policies and practices to reflect inclusion. While there are several challenges to overcome, organizational level changes in policies and practices in turn impact the workplace culture and the behaviours of individuals within, including the IENs themselves.

\section{Striving to achieve equity}

This third sub-process of two-way integration amplifies genuine openness and willingness to accept IENs as valuable contributors of knowledge and expertise. Having a grasp on the concept of equity, the employer organization recognizes the need to go beyond adopting inclusionary practices - that is, further than simply improving IENs' access to existing supports. Differential approaches, which are creative and responsive, are developed so that IENs may achieve equitable outcomes in terms of their professional growth. At the level of the IENs, such a context enables them to embrace the opportunities to progress on their leadership journey, albeit there many obstacles left to overcome.

This understanding of two-way integration shifts the focus from how IENs adapt or fit in to what IENs bring. Instead of being thought of as an 'IEN', colleagues think of her/ him as any other 'Canadian nurse'. Furthermore, a critical dimension to this third sub-process is the potential for IENs to influence practices based on the expertise they bring from having nursed internationally in other healthcare systems. This manager explains the shift in attention to the IENs' international experience: "For me, what's important is that they reach a point where... they become professionals with international experience as opposed to internationally educated professionals" (participant L005). Most IENs have worked in at least one country, if not in a few different countries before practicing in Canada. Some participants in this research acknowledge benefits of learning from IENs who have worked in other jurisdictions where healthcare systems are technologically more advanced, as well as from developing countries where IENs have had to nurse creatively with very limited resources. The net effect of how the diversity of experiences and talents of IENs enrich the organizational culture is described by this leader:

We think about the IENs fitting into the organization. But the true benefits of the internationally educated nurse, is not then just being a warm body and fitting into the organization but also what they bring to the organization, what they bring to patient care from the very fact that they are internationally educated and how they contribute and make our culture, not our culture in terms of Caucasian but I mean, our culture in terms of the organization, better (participant L026).
This valuing of IENs is aligned with SMH's core priority of delivering quality patient care. Its commitment to health equity is a core value and goal for its patients. Through its Centre for Research on Inner City Health, $\mathrm{SMH}$ has contributed to the growing body of evidence showing that marginalized people have the greatest health care needs, poorer access to services and the worst health outcomes. Staff and management collaborate in community initiatives with emphases on social determinants of health and issues of equity and access. One manager clearly articulates what equity means:

Equity is more than just treating everyone equally. In fact, it means doing more for some groups, because they start from a position of inequity in the first place...to bolster their position, you have to do more. (participant L025)

It is appreciated that concepts of health and illness are culturally defined, and that role of family, faith, spirituality, lifestyle including diet and exercise, may have varying interpretations and impacts on health outcomes. IENs' roles as cultural interpreters and knowledge brokers are acknowledged as important for colleagues to learn from and as part of becoming culturally competent in caring for diverse patients/families. This senior manager explains how IENs can help expose their colleagues to different ways of thinking about health and illness, and how the prevailing Canadian way may be limiting:

I think our IENs help us to be empathetic that there are other ways, not just the Canadian way of looking at treatment and...death, and even care of the body after, all of these things... I think, when I talk to IENs about these things, I become very sensitive to the fact that what you know is just what you know, it doesn't mean it's right. (participant L018)

By acknowledging that staff are part of the broader community, SMH focuses on addressing inequities within the workforce as well. Managers are open to differential supports for IENs as required, including mentoring, extended and/or tailored orientation programs. One peer/mentor provides the following example of differential support for a newly hired IEN:

We had a nurse from India... She was hired for one of the critical care units but they [her unit managers] ....sent her to our floor for a month, just to get her baseline, you know, just to get familiar with... Canadian nursing... They sent her to get trained on our floor for a month, which is general internal medicine, you get everybody there...So to me, that is great. You know, you just don't hire them [IENs] and then make them work in the critical care area...So that made the nurse feel very comfortable... when she was done a month... (participant M004) 
Encouragement from frontline managers is seen as vital for IENs to get involved on various committees or task groups. This peer/mentor explains how the managers' role is more than just making information available:

Well, they're posting what opportunity is there....and so everyone has equal opportunity to apply for that position. [But] the leadership encourages nurses to get involved...makes them [IENs] feel ...they can do the job...being approached. And then saying, "you would be good for this role... There is a position, or we have a committee, we are forming a committee, and would you like to be involved...?" (participant P002)

The workplace's commitment to IENs achieving equity has an important effect on their growth. Career development is an important area at $\mathrm{SMH}$, and they expect their managers to encourage IENs as much as their other staff to seek growth opportunities. An IEN states:

I stayed with St. Michael's because there's been ongoing support for my growth, if I chose to do that. It's a matter of my personal choice but it would be difficult if I wanted to do something and I'm not given an opportunity. That for me would be a problem. (participant I027)

Aside from supports for professional development, various opportunities to influence the workplace and practice are available at $\mathrm{SMH}$. IENs provide leadership in roles such as team leader, preceptor, mentor, educator, advanced practitioner, member on unit level or hospital wide committees and projects, as well as externally with professional associations and labour union. One IEN describes her leadership:

I'm a senior staff, others look up to me, like if there's an issue that needs to be addressed and they ask me [about] any recommendation or they need my help... I've been alternate for the last like five years as a charge nurse, unit leader...supervising the staff. (participant I015)

Another IEN speaks of how her graduate level research resulted in the adoption of new clinical protocols: "So
I got that changed, I was a change agent in that...now it's routine..." (participant 1012). The leadership's commitment to IENs' achieving their professional goals and aspirations extends beyond the confines of the workplace organization. A senior leader explains:

If a nurse wants to start in a general area and move to critical care, they can do that at St. Michael's. If they want to work in the community, they can do that. And so I look at integration beyond St. Michael's, I sometimes will actually even encourage them [IENs] to go to another organization that has programs and services that are aligned with their true passion and vision. So integration means at the end of the day, that people get up and go to work where they feel they can maximize their full potential (participant L018).

Senior leadership is consciously behaving in ways that are strategic and systemic, striving for IENs to achieve equity at the wider system level. One senior leader states:

It's not just "okay, now they're here and they're fully working, and my job is done". It's now how do they contribute back to the cause of IENs... as a leader you have to understand the national frame, the provincial frame and see that this is...just part of your job...I've always looked for opportunities...(participant L018)

This third sub-process of two-way integration emphasizes the employer's recognition of the talents and expertise that IENs bring. In addition to their diverse ethno-racial backgrounds and life experiences, IENs' international nursing experiences from other healthcare systems are also valued. Given that many IENs are in potential positions of inequity, there is a proactive approach to providing differential supports for participation in various roles and development of their full leadership potential. Although there are challenges, the organization's capacity to apply the concept of equity to IENs' integration is enhanced by its fundamental commitment to health equity for its diverse patients and community. Figure 1 summarizes the three sub-processes of the two-way integration.

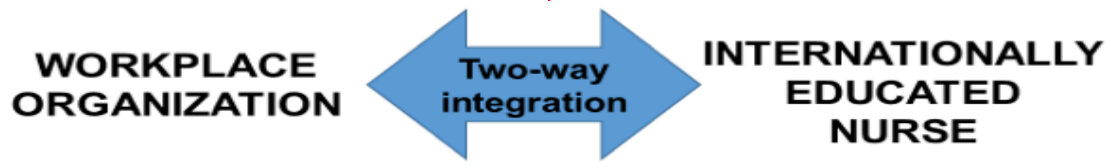

1. Respecting diversity and difference

Organization prioritizes workforce diversity in response to patients' needs: supports IEN with 'fitting in'. IEN adapts to differences in nursing practice and culture; now being 'Canadian'.

II. Adopting inclusive practices

Organization-wide, systematic efforts address exclusion through policy and procedural changes and education to sustain a safe, inclusive workplace
culture. IEN redefines, refines own values; is at ease with own identity

III. Striving to achieve equity

Workplace commitment to equity for IENs reflected in accountability systems. IEN's talents and expertise valued; organization opens up to being influenced by the IEN's international experiences. Differential supports for IEN are in place. IEN progresses on leadership journey. 


\section{Discussion}

Workplace integration of IENs as a two-way process and outcome is new to nursing. $[13,24]$ However, within the social sciences, notions of two-way integration of immigrants and refugees into their host communities have been part of the discourse for some time now. Definitions in immigrant and refugee studies describe integration as a mutual, twoway process between the new home or host society and the newcomers.[25-27] In Canada, the 1971 Multicultural Act and the federal Charter of Rights and Freedoms has provided the framework for the Integration Program which has the intent of encouraging a process of mutual accommodation and adjustment by both newcomers and the larger society. [28] Ley[29] explains that 'integration' attempts to signify a break with assimilation, particularly in a Canadian context where the federal policy "institutionalizes not only respect for difference but also the rights of being different". [p7] Despite the policy and dialogue, there is concern that program investments have been primarily focused on initial stages of adaptation and in promoting interactions among newcomers rather than with established immigrants or those born in Canada. $[27,30]$

Given this backdrop, the case organization, $\mathrm{SMH}$, is indeed exemplary in its efforts to create a workplace environment where two-way integration of IENs is discussed and demonstrated in multiple ways. Perhaps being situated in the heart of Canada's most ethno-racially diverse city makes it easier since issues of equity, diversity and inclusion are 'in your face' and inertia is not an option. Despite its successes, $\mathrm{SMH}$ has experienced several challenges which are also identified by other studies. These tensions are discussed next and include: respecting diversity but conforming; increasing inclusion broadly at the risk of reinforcing marginalization for "others" and striving for equity but not being accountable.

\section{Respecting diversity but conforming}

Within the context of the first sub-process of respecting diversity and difference, various examples of difficulties related to workplace culture and communication skills of IENs were provided by participants. One significant challenge that IENs face is learning to communicate in Canadian English, especially the appropriate use of idioms, jargon, slang and acronyms. This has been widely documented in the literature. $[3,31]$ Workplace culture and communication challenges remind IENs of their foreignness and lack of belonging, which often increase their sense of vulnerability. Difference based on ethno-racial backgrounds has been the subject of several studies on racism in nursing.[18,19,32-41]
In a study of China-educated nurses practicing in Australia, negative meanings such as incompetence were ascribed to difference.[41] Etowa, et al.[19] found that Black nurses in Nova Scotia reacted to such experiences with a strong sense of insecurity and isolation. According to Etowa et al.'s[19] "theory of surviving on the margins"(p177), intense efforts on the part of IENs trying to adapt to become "Canadian" are appeasing strategies. These are in direct response to highly pressurized experiences and the strong desire to ensure they are liked by those are in the "centre" or who have more power.

At the level of the workplace, Allen[42] explains that sometimes the focus on diversity is primarily one of "add colour and stir".[p66] It is simply implied that a bunch of "other" people need to be taken into the mainstream organization.[42] Within such a context, Lukes'[14] overt dimension of power may be at play where processes are 'open' to everyone but there is little or no regard for IENs requiring any differential supports to integrate in the workplace. Although the organizational priority of workforce diversity is impressive, if policies and practices are left as neutral, they maintain the privilege of those who have traditionally held power and can have adverse impacts on individuals who are different from the dominant group. $[43,44]$ This leads us to the sub-process of adopting inclusive practices where there are potential challenges as well.

Increasing inclusion broadly at the risk of reinforcing marginalization for "others"

Inclusion refers to the systemic nature of an organization, and to achieve it, workplaces must create policies and practices that recognize more than one view and that signal importance of learning from differences.[43] Healthcare organizations are faced with multiple and competing priorities. Sustaining inclusive practices requires thoughtful and measured efforts; gaining buy-in from all levels of leadership across large organizations can be an issue. When an inclusive definition of diversity, in which all differences (e.g. gender, age, race, socio-economic status, sexual orientation, abilities, etc.) is used to appeal to a broad audience, the dialogue related to race is not made explicit. This may be equated to Lukes' [14] covert face of power whereby leaving race 'off the agenda' results in continued marginalization and other painful experiences for racialized individuals. [45] Thus, there is a risk that organizational inclusion is in disguise whereby IENs, or other peoples 'of difference' are invited in, but are the only ones made to change and not the workplace.

Inclusive organizational environments are conducive to 
encouraging self-reflection, self-determination and critical analysis at the individual staff level.[46-48] To be inclusive, it is recognized that needs for resources within the organization may vary among people, at various times and according to capabilities.[43] Additionally, a safe climate to ask critical questions that reveal decision-making mechanisms for how 'rules' in the form of policies, written and unwritten, have come about and how they affect different groups should be cultivated continuously within an inclusive framework.[49] In this sense the organization is open to continuous improvements to existing structures or systems that may be creating social closure or are exclusionary for specific groups.[50]

\section{Striving for equity but not being accountable}

There are multiple challenges in realizing the third subprocess of two-way integration of striving to achieve equity. At the core of these challenges is likely Lukes' (14) latent face of power, the most difficult for the IEN to identify as her/ his very wants have been influenced. Firstly, as many experts in immigrant and refugee studies have stated, commitment to equity without accountability is inadequate. $[43,51]$ Well intentioned organizations often fail to put accountability mechanisms in place. Specific targets and measures, both quantitative and qualitative, are essential if organizations are serious about acting on their value of achieving equitable outcomes for IENs.[43,51] The lack of accountability systems can also suggest that the link between the priority of integration of IENs and the organization's core business of delivering quality care has not been clearly established. The resulting scenario is where IENs' successful integration experiences of progressing on their leadership journey are limited to anecdotes from certain pockets of the organization.

Another related challenge has to do with difficulties in understanding the concept of equity. To be equitable, workplace policies or systems must be considerate of the need for differential supports for diverse staff.[43] Without continuous education and dialogue, managers could remain unclear (and unconvinced) about the importance of differential approaches for supporting IENs. Managers can become preoccupied with how offering of differential supports to IENs may be favoritism to the extent that IENs themselves may also start to adopt this perspective and resist the necessary assistance.

Finally, the lack of comfort in self-promotion is further compounded by the preoccupation for the IENs to fit in and adapt to Canadian nursing practice. The intense pressures of practicing as 'Canadian nurses' leaves little or no disposable time or energy for IENs to be analytical about the relevance of their international experiences to their Canadian nursing practice. If structured mechanisms are not in place to draw out IENs' professional insights from international contexts, Canadian nursing practice get signalled as the "gold standard". [52] The resulting effect of Lukes' [14] latent face of power manifests here such that IENs may internalize that their practice is inferior and requires 'fixing'. IENs hold back or refrain from sharing and utilizing their international experiences in the solving of local nursing problems.

\section{Implications}

Given various challenges at the individual IEN and the organization levels, it is reasonable that facilitating workplace integration is a continuous work in progress. Based on the experiences of $\mathrm{SMH}$ and experts, the following insights are summarized to assist other organizations who are also striving for two-way integration of IENs:

- Diversity alone does not bring about an inclusive culture. Minors et al.[43] state that all the characteristics of an organization, from its structure, decision-making processes, communication vehicles, and even to its mission, are based on assumptions about individuals and groups that comprise the dominant norm.

- Inclusive organizational cultures can exist without diversity within their workforce.[53] However, it is argued that to maximize organizational achievement, both diversity and inclusion are essential.[53] With increasing diversity within the staff and patient populations, challenging of assumptions and establishing new ones is necessary to promote inclusive practices. [43]

- Equity is a difficult concept to grasp - both in terms of health equity for patients and workplace equity for IENs. Managers and staff need regular opportunities to practice applying the 'equity lens' when reviewing policies and procedures and in planning programs. Continuous reminders that not everybody starts from the same position are necessary. The perceptions of needing to treat everyone equally or having that as a goal, could in fact reinforce status quo and systemic barriers. Differential supports for IENs are not unreasonable to achieve equitable outcomes.

- Measuring disparities and improvements are cornerstones of all equity strategies. Establishing meaningful metrics, both quantitative and qualitative for different populations is key. $[43,51,54]$ This is equally true of workplace equity for IENs, as it is of healthy equity of patients. 
- Striving to achieve equity is more than having respect for diversity and difference and for adopting inclusive practices. Saloojee[44] describes equity as follows: "it extends beyond bringing the 'outsiders' in, or notions of the periphery versus the centre. It is about closing physical, social and economic distances separating people, rather than only about eliminating boundaries or barriers between us and them".(p.ix) Accountability measures and mechanisms are imperative.

- Benefits of two-way integration are beyond IENs. They extend to patients and families as well as various segments of the workforce, including Canadian educated and racialized nurses and other groups of internationally educated professionals. To prevent regression because of downsizing or budget cuts, a business case for IEN integration is required, with close alignment to the organization's core priority of delivering quality care.[55] Like in the business/trade sectors, nursing and healthcare leaders should recognize that as immigrants, IENs can be catalysts for innovation and new connections.[56] In this valuing context opportunities are cultivated for IENs to influence and shape nursing practice. [7]

\section{Limitations}

This research is a qualitative, instrumental case study involving a single organization within a specific community context. There are no expectations of replicating this study as this is a foundational work exploring the concept of workplace integration of IENs. However, it is conceivable that this study may lead to a program of research with multiple sites. The use of $\mathrm{SMH}$ as an exceptional case may be a limitation especially if one is interested in a deeper understanding of how social closure operates to exclude IENs from organizational processes. A thick description of the organizational context is provided so that the interested readers can reach a conclusion about transferability to other similar situations.

\section{Conclusion}

Recognizing that the notion of workplace integration of IENs is a two-way process requiring efforts at both the individual IEN and the organization levels is the unique contribution of this research. The one-sidedness of IEN integration found in the nursing literature to date is problematic as it places the burden of responsibility solely on the IEN. This research highlights that organizational efforts to facilitate IEN integration have the potential for broader benefits with respect to quality care for patients and their families, as well as positive changes for other groups of staff who may also be in positions of inequity. The case study approach and the selection of an exemplar organization have made it possible to gather rich insights about how two-way integration is experienced and explained by participants in multiple ways. While some will quell at the emerging ideas, it is our hope that nursing leaders will be incited to reflect and assess approaches to workplace integration of IENs within their organizations. Thoughtful and deliberate action by everyone will maximize the talents and experiences that IENs bring for the benefit of their patients and communities.

\section{References}

1.Freeman M, Baumann A, Fisher A, Blythe J, Akhtar-Danesh $N$. Case study methodology in nurse migration research: An integrative review. Applied Nsg Research 2012; 25: 222-228.

2.Newton S, Pillay J, Higginbottom G. The migration and transitioning experiences of internationally educated nurses: A global perspective. Journal of Nursing Management 2012; 20(4): 534-550. doi: 10.1111/j.1365-2834.2011.01222.x; 10.1111/j.1365-2834.2011.01222.x

3.Xu Y. Transition as a salient area of inquiry in healthcare: Asian nurses working in western countries. Home Health Care Management \& Practice 2007; 19(6): 485-487. doi:10.1177/1084822307304249

4.Zizzo K., Xu Y. Post-hire transitional programs for international nurses: A systematic review. The Journal of Continuing Education in Nursing 2009; 40(2): 57-64.

5.Tilley C. Support for internationally educated nurses transitioning into practice: An integrative literature review 2007; Unpublished manuscript. Victoria, B.C: School of Nursing, University of Victoria.

6.Primeau M, Champagne F, Lavoie-Tremblay M. ForeignTrained Nurses' Experiences and Socioprofessional Integration Best Practices - An Integrative Literature Review. The Health Care Manager 2014; 35 (3): 245-253.

7.Raghuram P. Interrogating the language of integration: the case of internationally recruited nurses. Journal of Clinical Nursing 2007; doi:10.1111/j.1365.2702.2007. 02097.x

8.Ramji Z, Etowa J. Current perspectives on integration of internationally educated nurses into the healthcare workforce. Humanities and Social Sciences Review 2014; CD ROM:ISSN: 2165-6258: 03 (03):225-233

9.Adams E, Kennedy A. Positive practice environments Key considerations for the development of a framework to support the integration of international Nurses 2006; Geneva: 
International Centre on Nurse Migration.

10.Canadian Health Human Resources Network (CHHRN). Knowledge synthesis - internationally educated health professionals: A review of the Canadian literature 2013; Ottawa: CHHRN.

11.Covell CL, Neiterman E, Bourgeault I. A scoping review of the literature on internationally educated nurses in Canada: Mapping a research agenda. Canadian Journal of Nursing Research 2014; 46(3): 26-45.

12.Salma J, Kathleen MH, Ogilvie L. Career advancement and educational opportunities: Experiences and perceptions of internationally educated nurses. Canadian Journal of Nursing Leadership 2012; 25(3): 56-69.

13.Ramji Z, Etowa J. Workplace integration: Key considerations for internationally educated nurses and employers. Administrative Sciences 2018; 1-18. doi:10.3390/ admsci8010002.

14.Lukes S. Power: A radical view. New York: Palgrave MacMillan, 2005.

15.Gaventa J. Power and powerlessness - quiescence and rebellion in an Appalachian valley. Urbana: University of Illinois Press, 1980.

16.Sadan E. Empowerment and Community Planning: Theory and Practice of People-Focused Social Solutions. Tel Aviv: Hakibbutz Hameuchad Publishers [in Hebrew, translated to English by Richard Flantz], 1997.

17.Freire P. Pedagogy of the Oppressed. New York, NY: Herder \& Herder, 1971.

18.Das Gupta T. Real nurses and others - Racism in nursing. Nova Scotia: Fernwood Publishing, 2009.

19.Etowa J, Sethi S, Thompson-Isherwood R. The substantive theory of surviving on the margin of a profession. Nursing Science Quarterly, 2009 22(2): 174-181.

20.Hagey R, Choudhry U, Guruge S, Turrittin J, Collins E, Lee R. Immigrant nurses' experience of racism. Journal of Nursing Scholarship, 2001; 33(4):389-394.

21.Stake RE. The art of case study research. Thousand Oakes, CA: SAGE Publications, 1995.

22.St. Michael's Hospital. 2010 Health Equity Refresh Report - Quality Healthcare for All - Prepared for the Toronto Central Local Health Integration Network. Toronto, ON: St. Michael's Hospital, 2010.

23.Braun V, Clarke V. Using Thematic Analysis in Psychology.
Qualitative Research in Psychology, 2006; 3(1):79-89.

24.St-Pierre I, Covell CL, Primeau M, Kilpatrick K, Ndengeyingoma A, Tchouaket E. Factors that Support the Integration and Retention of Internationally Educated Nurses in the Canadian Healthcare Systems. Ottawa: Health Canada, 2015.

25. Hyndman J. Research summary on resettled refugee integration in Canada. Toronto, ON: Centre for Refugee Studies, York University, 2011. Retrieved from http://www. unhcr.ca/resources/4e4b77842.pdf

26.UNHCR. Executive committee conclusion on local integration 2005, No. 104 (LVI). Retrieved from http://www. unhcr.org/4357a91b2.html

27.Wong W, Poisson Y. From immigration to participation: A report on promising practices in integration. Ottawa, ON: The Public Policy Forum, 2008. Retrieved from http://www. hippycanada.ca/downloads/Immigration.pdf

28.Citizenship \& Immigration Canada. Annual Report, 2013. Retrieved from http:/www.cic.gc.ca/english/resources/ publications/annual-report-2013/section4.asp\#a3

29.Ley D. Post-Multiculturalism? Working Paper No. 05-18. Research on Immigration and Integration in the Metropolis. Vancouver: Vancouver Centre of Excellence, 2005.

30.Omidvar R, Richmond T. Immigrant settlement and social inclusion in Canada. Toronto, ON: The Laidlaw Foundation, 2003. Retrieved from www.laidlawfdn.org/working-paperseries-social-inclusion

31. Blythe J, Baumann A, Rheaume A, Mclntosh K. Nurse migration to Canada: Pathways and pitfalls of workforce integration. Journal of Transcultural Nursing: Official Journal of the Transcultural Nursing Society / Transcultural Nursing Society, 2009; 20(2): 202-210. doi:10.1177/1043659608330349

32.Allan H. Mentoring overseas nurses: Barriers to effective and non-discriminatory mentoring practices. Nursing Ethics, 2010; 17(5): 603-613. doi:10.1177/0969733010368747

33.Batnitzky A, McDowell L. Migration, nursing, institutional discrimination and emotional/affective labour: Ethnicity and labour stratification in the UK National Health Service. Social \& Cultural Geography, 2011;12(2): 181-201. doi:10.1 080/14649365.2011.545142

34.Dicicco-Bloom, B. The racial and gendered experiences of immigrant nurses from Kerala, India. Journal of Transcultural Nursing: Official Journal of the Transcultural Nursing 
Society / Transcultural Nursing Society,2004, 15(1), 26-33. doi:10.1177/1043659603260029

35. Hawthorne L. The globalisation of the nursing workforce: Barriers confronting overseas qualified nurses in Australia. Nursing Inquiry, 2001, 8, 213-229.

36.Kawi J, Xu Y. Facilitators and barriers to adjustment of international nurses: An integrative review. International Nursing Review, 2009, 56(2), 174-183. doi: 10.1111/j.14667657.2008. 00705.x

37.Magnusdottir H. Overcoming strangeness and communication barriers: a phenomenological study of becoming a foreign nurse. International Nursing Review, 2005, 52, 263-269.

38. Mapedzahama V, Rudge T, West S, Perron A. Black nurse in white space? Rethinking the in/visibility of race within the Australian nursing workplace. Nursing Inquiry, 2012,19, 153-164.

39.Pizer CM, Collard AF, James SM, Bonaparte BH. Nurses' job satisfaction: Are there differences between foreign and US-educated nurses? Image--the Journal of Nursing Scholarship, 1992, 24(4), 301-306.

40.Turrittin J, Hagey R, Guruge S, Collins E, Mitchell M. The experiences of professional nurses who have migrated to Canada: cosmopolitan citizenship or democratic racism? International Journal of Nursing Studies, 2002, 39, 655-667

41.Zhou Y, Windsor C, Theobald K, Coyer F. The concept of difference and the experience of china-educated nurses working in Australia: A symbolic interactionist exploration. International Journal of Nursing Studies, 2011, 48(11), 14201428. doi: 10.1016/j.ijnurstu.2011.05.003

42.Allen DG. Whiteness and difference in Nursing. Nursing Philosophy, 2006, 7, 65-78.

43. Minors A. Creating anti-Racist organizations. The university to poly-versity series on anti-oppression organization change. Toronto: Arnold Minors \& Associates. 2015.

44.Saloojee A. Social inclusion, anti-racism and democratic citizenship. Toronto: The Laidlaw Foundation. 2003.

45.Kersten A. Diversity management. Journal of Organizational Change Management, 2000, 13(3), 235-248. doi: 10.1108/09534810010330887

46. Getty GA.The journey between western and indigenous research paradigms. J Transcultural Nsg, 2010, 21(1), 5-14.

47.Manias E, Street A. Possibilities for critical social theory and Foucault's work: A toolbox approach. Nursing Inquiry, 2000, 7(1), 50-60.

48. Roberts S. Oppressed group behavior: implications for nursing. Advances in Nursing Science, 1983, 21-30.

49.Nussbaum M. Women's capabilities and social justice. Journal of Human Development, 2005, 1(2), 219-247. doi:10.1080/14649880020008749

50.Roscigno V, Mong S, Byron R Tester G. Age discrimination, social closure and employment. Social Forces, 2007, 86(1), 313-334.

51.Galabuzi G. Employment equity as "felt fairness": The challenge of building an inclusive labour market. Canadian Diversity, 2012, 9(1), 22-28.

52.Etowa J, Debs-Ivall S, Conners E. Engaging with racism: An opportunity to advance nursing practice. The International Journal of Health, Wellness, and Society, 2015, 5(2), 1-13.

53.Bormann T, Woods S. A framework for building inclusive organizations. New York: The Workplace Diversity Network, Cornell University. 1999. Retrieved from www.ilr.cornell. edu/wdn/upload/paper2.pdf

54.Toronto Central Local Health Integration Network (TCLHIN). Toronto Central LHIN stakeholder consultation report. Toronto: Toronto Central LHIN. 2011.

55.Turner T. Diversity 2020:12 Upcoming equity, diversity and inclusion trends and issues. Toronto: Turner Consulting Group Inc. 2014.

56.Parkouda M. The immigrant advantage: Leveraging immigrants for innovation and competitiveness. Presented at Canadian Immigration Conference 2014: Enhancing Canada's Immigration System — from Invitation to Integration. Toronto, ON: The Conference Board of Canada.2014.

Acknowledgements

Financial support for this postdoctoral research was obtained through the University of Ottawa's Faculty of Graduate and Postdoctoral Studies and the Nursing Leadership Network of Ontario. The authors would also like to express thanks to Ms. Betsy Kappel, Dr. Ivy Bourgeault, and Dr. Rani Srivastava. Their generous and constructive feedback has been critical in developing the concepts and ideas contained in this paper.

To contact the authors:

Zubeida Ramji, RN, PhD

Z. Ramji Associates Inc.,

141 Sandyhook Square,

Toronto, Ontario, M1W 3N6;

Canada

email: zramj091@uottawa.ca 